\title{
BMJ open HIV incidence and prevalence among cohorts of women with higher risk behaviour in Bloemfontein and Rustenburg, South Africa: a prospective study
}

\author{
Paul J Feldblum, ${ }^{1}$ Mary H Latka, ${ }^{2}$ Johann Lombaard, ${ }^{3}$ Candice Chetty, ${ }^{2}$ \\ Pai-Lien Chen, ${ }^{1}$ Connie Sexton, ${ }^{1,{ }^{*}}$ Shelly Fischer ${ }^{1}$
}

To cite: Feldblum P, Latka M, Lombaard J, et al. HIV incidence and prevalence among cohorts of women with higher risk behaviour in Bloemfontein and

Rustenburg, South Africa: a prospective study. BMJ Open 2012;2:e000626. doi:10.1136/

bmjopen-2011-000626

- Prepublication history for this paper is available online. To view these files please visit the journal online (http:// dx.doi.org/10.1136/ bmjopen-2011-000626)

Received 1 December 2011 Accepted 16 January 2012

*Now at the US Centers for Disease Control and Prevention.

This final article is available for use under the terms of the Creative Commons Attribution Non-Commercial 2.0 Licence; see http://bmjopen.bmj.com

${ }^{1} \mathrm{FHI}$ 360, Research Triangle Park, USA

${ }^{2}$ The Aurum Institute, Johannesburg, South Africa ${ }^{3}$ JOSHA Research, Bloemfontein, Free State, South Africa

Correspondence to Dr Paul Feldblum; pfeldblum@fhi360.org

\section{ABSTRACT}

Objectives: The primary objective was to measure HIV incidence in two prospective cohorts of HIV-negative women. Secondary objectives included measuring pregnancy rates and participant retention rates.

Design: Cross-sectional HIV screening of women selected for higher risk behaviours, with a subsequent prospective study of uninfected women, followed monthly for up to 6 months.

Setting: Clinics established for research purposes in Bloemfontein and Rustenburg, South Africa.

Participants: The authors enrolled women 18-35 years old and presumed at higher risk of sexual acquisition of HIV as indicated by self-reported sexual behaviour or recent sexually transmitted infection symptoms. In Bloemfontein, 1364 women were screened, 1154 were eligible for HIV testing and 1145 agreed to be tested. The prospective study enrolled 401 HIV-negative women. In Rustenburg, 946 women were screened, 540 were eligible and underwent HIV testing and 223 HIV-negative women entered the prospective study.

\section{Primary and secondary outcomes: Baseline} prevalences of HIV infection and HIV incidence rates in the prospective cohorts, according to a double rapid test algorithm with a third rapid test for discrepant or indeterminate results. Pregnancy prevalences and pregnancy incidence rate in Bloemfontein. Participant retention rates in the prospective cohort until the study end.

Results: In Bloemfontein, 1145 women were tested, 391 entered follow-up and $92.3 \%$ of participants completed six study visits. In Rustenburg, 540 women were tested, 194 entered follow-up and retention up to the point of early study termination was $88.6 \%$. Overall HIV prevalence was $21.2 \%(95 \% \mathrm{Cl}$ $18.9 \%$ to $23.6 \%)$ in Bloemfontein and $23.5 \%(95 \% \mathrm{Cl}$ $19.9 \%$ to $27.1 \%$ ) in Rustenburg. Overall HIV incidence was $5.5 / 100$ person-years $(95 \% \mathrm{Cl} 2.5$ to 10.4$)$ in Bloemfontein and 3.0/100 person-years (95\% $\mathrm{Cl} 0.4$ to 10.8) in Rustenburg. Cross-sectional pregnancy prevalences were $6.5 \%$ in Bloemfontein and $8.6 \%$ in Rustenburg.

\section{ARTICLE SUMMARY}

Article focus

- Primary objective: measure HIV incidence and prevalence among women in two prospective cohorts selected for higher risk behaviours.

- Secondary objective 1: measure pregnancy rates.

- Secondary objective 2: measure retention rates.

Key messages

- We measured the HIV burden among women selected to be at higher risk in two smaller South African cities, while training new clinical research groups there.

- HIV seroprevalence was $21.2 \%$ in Bloemfontein and $23.5 \%$ in Rustenburg.

- HIV incidence rates were $5.5 / 100$ person-years in Bloemfontein and $3.0 / 100$ person-years in Rustenburg.

Study strengths

- Longitudinal design with high retention rates.

- Highly accurate classification of the HIV outcome, using multiple rapid tests monthly, confirmed by later PCR testing.

- Testing of stored baseline specimens to pinpoint seroconversion and rule out acute prestudy infections.

- Direct prospective measurement of HIV infection circumvented the lingering issues with the accuracy of cross-sectional incidence estimation.

Study limitations

- Unknown validity of self-reported sexual behaviours.

- Relatively small study size precluded multivariable Cox regression analysis.

- Selective recruitment means that the prevalence and incidence figures are not representative of women in these two cities.

- The lack of sexually transmitted infection diagnostic testing precluded evaluating associations between HIV and other infections. 
Conclusions: The authors observed substantial HIV incidence rates in both cohorts. Vigorous prevention efforts are needed in these smaller cities.

\section{INTRODUCTION}

HIV/AIDS continues to exact a massive toll on the health, economics and political infrastructure of communities around the world. In sub-Saharan Africa, the most heavily affected region, an estimated 22.4 million people were reported to be living with HIV in 2008. While recent prevention trials assessing male circumcision, antiretroviral gel use and pre-exposure prophylaxis have shown significant promise, ${ }^{2-5}$ studies to corroborate these results and to evaluate new prevention methods will be required. To meet the ongoing need for research sites in countries with substantial HIV burden, FHI 360's Site Identification and Development Initiative (SIDI) undertook the development of previous research naive sites in six countries to conduct future HIV prevention research.

The SIDI team partnered with groups in two South African cities for clinical research development: Bloemfontein in the Motheo District of Free State Province and Rustenburg in the Bojanala District of Northwest Province. District-level HIV prevalence rates among antenatal women aged 15-49 years were $27.8 \%$ and $34.9 \%$, respectively, in 2009; provincial HIV prevalence rates were $30.1 \%$ (Free State) and 30\% (Northwest). ${ }^{6} \mathrm{HIV}$ incidence rates for both sites had not been measured.

The goal of the SIDI project was to develop HIV prevention research capacity and to determine the suitability of each site to implement future HIV prevention clinical trials. Each site conducted its own prevalence and incidence study using a similar protocol. The primary objective of each study was to measure HIV incidence in a prospective cohort of HIV-negative women at each study site. Secondary objectives included measuring pregnancy rates and participant accrual and retention rates in the cohort.

\section{METHODS}

\section{Study design and recruitment}

The study comprised cross-sectional screening with HIV testing, followed by a prospective cohort study with up to six monthly follow-up visits. In Bloemfontein, the study was conducted at the JOSHA Research Centre. Bloemfontein and the surrounding Mangaung municipality comprise a population of approximately 600000 people. JOSHA is an independent clinical research facility that can draw participants from affiliated general practices as well as from cooperating government clinics. Participants for the cross-sectional screening were recruited using methods developed based on the feedback from community engagement activities led by the MangaungUniversity Community Partnership Program (MUCPP) and input from key stakeholders in the community.
Recruitment methods included (1) recruitment from HIV counselling and testing, sexually transmitted disease and family planning clinics; (2) targeted outreach activities in the community and (3) referrals from other clinics. Participants for the prospective study were recruited from among the HIV-negative participants in cross-sectional screening.

In Rustenburg, with a population estimated at 350000 in the 2001 census but which has grown considerably since then, the study was conducted at The Aurum Institute's Rustenburg Research Centre located downtown. The Rustenburg economy is largely driven by the platinum mining industry and is home to a large, diverse and growing influx of residents attracted to the area for work in the mining industry. The Aurum Institute (formerly the Aurum Institute for Health Research) is a non-profit research organisation that began activities in March 2007 to prepare the Rustenburg Municipality to engage in epidemiologic studies and future clinical research trials. Participants for the cross-sectional screening were recruited from a variety of clinic-based and community settings, which included an emphasis on local colleges. To facilitate recruitment and enrolment for cross-sectional screening, the protocol was amended twice late in the study to add respondent-driven sampling for recruitment (not inference) $\operatorname{purposes}^{7}$ and to liberalise the sexual behaviour eligibility criterion. Both men and women were recruited for cross-sectional screening in Rustenburg, but only HIV-negative women were invited to participate in the prospective study as there was a separate male cohort study there.

\section{Study procedures}

Following the informed consent process and assignment of a participant number, volunteers were considered enrolled. Enrolled participants were screened for eligibility for cross-sectional testing using a demographic and HIV risk factor questionnaire. Recruitment and screening staff at both sites were blind to the specific behavioural eligibility criteria to avoid the spread of information through the community and to prevent volunteers from altering self-reported behaviours to gain access to the study. All participants were asked the full questionnaire regardless of their initial answers.

Only eligible women were tested. Eligibility for HIV and pregnancy testing was determined by age (18-35 at the time of screening) and presumed higher risk of sexual acquisition of HIV as defined by at least one of the following self-reported criteria in the past 3 months: (1) treatment for, diagnosis of or signs/symptoms of a sexually transmitted infection (STI); (2) vaginal or anal intercourse with more than one partner (unprotected intercourse in Rustenburg only); (3) vaginal or anal intercourse with a new sexual partner (unprotected intercourse in Rustenburg only) or (4) sex with an HIVinfected partner who is not using antiretroviral therapy. Near the end of the Rustenburg study, we revised these criteria to remove the requirements for multiple partners and unprotected sexual acts for women, as we 
suspected that we were screening out many higher risk women and that condom use reporting was not accurate. Participants deemed eligible underwent HIV testing within the context of HIV pretest and post-test counselling in accordance with national guidelines. Women also had urine pregnancy tests. Results of both tests were provided to participants during their visits.

Women were eligible to enter the prospective cohort study if they met the cross-sectional age and behavioural criteria, were HIV negative and pregnancy negative and were not planning to relocate from the area in the near future. The target prospective study size was the first 400 eligible women at each site, followed monthly for up to 6 months. At each monthly visit, participants provided updated medical and behavioural information, underwent HIV and pregnancy testing, received syndromic management for STIs (as needed), were counselled on HIV risk reduction practices and received free condoms.

HIV testing was performed on finger prick blood samples using a double rapid test algorithm with the Abbott Determine HIV-1/2 and Trinity Biotech UniGold run in parallel. Two positive results were deemed positive and two negative results were deemed negative, which exceeded national HIV testing guidelines. Discrepant or indeterminate samples were tested with a third rapid test, SD BIOLINE HIV-1/2 3.0, as a tiebreaker. Pregnancy testing was performed on urine samples using the Quidel QuickVue hCG-Combo test. All tests were run according to the manufacturers' specifications.

Participants who tested HIV positive at screening or during the cohort study were referred for care in accordance with local guidelines. In addition, those participants who seroconverted during the prospective phase were intensely counselled about secondary HIV prevention in the acute infection period and in Rustenburg were invited to join a seroconvertor cohort with continued close monitoring. Participants who were found to be pregnant were referred for antenatal care. Participants who became pregnant during follow-up continued to make study visits.

\section{Ethical considerations}

The protocols, informed consent forms, participant education and recruitment materials, case report forms and compensation amount for visits (50 rand) were reviewed and approved by the FHI 360 ethics committee and the local ethics committees, the University of Free State ethics committee for Bloemfontein and the University of KwaZulu Natal Biomedical Research Ethics Committee for Rustenburg.

We obtained written informed consent from each participant prior to enrolment and data collection. Written consent was also obtained for long-term specimen storage and possible future testing, although consent for specimen storage was not required for study participation. Informed consent forms were available in English and were translated into local languages, Sesotho (Bloemfontein) and Sestwana and IsiXhosa
(Rustenburg), to enhance comprehension. Volunteers had their choice of language for the informed consent process, which was witnessed and verified by a non-study staff person for illiterate or low-literate participants.

\section{Statistical analysis}

All eligible participants were included in the data analysis. For each site, we summarised participants' sociodemographic characteristics, self-reported STI symptoms and HIV risk behaviours at baseline for cross-sectional screening participants and those women who entered the prospective study. We calculated baseline prevalences of HIV infection and pregnancy among women, along with $95 \%$ CIs. We used univariate and multivariable logistic regression analysis to evaluate the association between risk factors and prevalent HIV infection.

The incidence rate was calculated as the number of post-enrolment HIV seroconversions divided by the person-years (PY) of observation accumulated by the cohort. We calculated $95 \%$ CI using exact methods under the assumption that the number of HIV infections follows a Poisson distribution. We used proportional hazards regression to evaluate the associations between incident HIV infection and possible HIV risk factors including time-dependent participant characteristics and time-varying risk behaviours and self-reported STI symptoms. Due to the small number of HIV seroconversions, we did univariate proportional hazards regression only. Data analyses were performed using SAS V.9.2.

\section{RESULTS}

\section{Eligible populations}

In Bloemfontein, the cross-sectional screening enrolled 1364 women between February and October 2009. Of the 1364 enrolled women, 1154 were determined to be eligible for testing and 1145 (84\% of enrollees) chose to be tested in cross-sectional screening. Virtually all of the women who were screened out from cross-sectional testing failed to satisfy the behavioural or STI risk criteria. The prospective study enrolled $401 \mathrm{HIV}$-negative women volunteers from screening. Of those 401 women, 399 were determined to be eligible and 391 entered followup and were tested. $92.3 \%$ of participants completed six study visits, for a median 5.6 person-months of observation and a total 164.4 PY.

In Rustenburg, cross-sectional screening enrolled 946 women and 968 men between November 2008 and December 2009. Data on the male participants are not presented here. Of the 946 enrolled women, 540 (57\%) were eligible and underwent testing in cross-sectional screening; virtually all of the women who were screened out from cross-sectional testing did not satisfy the behavioural or STI risk criteria. The prospective study enrolled 223 HIV-negative women volunteers, of whom 194 yielded HIV test data. In Rustenburg, many women screened out from the prospective study due to plans to re-locate. Completion of follow-up in the prospective study was hindered when it was ended prematurely due to funding shortfalls, but retention in follow-up up to the 
point of study termination was $88.6 \%$ with a median 4.1 person-months of observation and a total $67.1 \mathrm{PY}$.

\section{Participant characteristics}

Large percentages of participants in cross-sectional screening at both sites were $\leq 21$ years, had a high schoollevel education, were not employed, were unmarried and lived with family members (table 1). About one-third of the women were using an effective contraceptive at each site (hormonal method or IUCD: $38.3 \%$ in Bloemfontein and $32.9 \%$ in Rustenburg; table 1). STI testing was not done; self-reported STI signs/symptoms at baseline ranged from $2 \%$ to $15 \%$ prevalence in Bloemfontein and from $22 \%$ to $37 \%$ in Rustenburg.

Table 1 Participant features $(\mathrm{N}(\%))$ of eligible women at inception of cross-sectional screening and prospective study in Bloemfontein and Rustenburg sites

\begin{tabular}{|c|c|c|c|c|}
\hline & \multicolumn{2}{|l|}{ Bloemfontein } & \multicolumn{2}{|l|}{ Rustenburg } \\
\hline & $\begin{array}{l}\text { Cross-sectional, } \\
\mathrm{N}=1154\end{array}$ & $\begin{array}{l}\text { Prospective, } \\
\mathrm{N}=399\end{array}$ & $\begin{array}{l}\text { Cross-sectional, } \\
\mathrm{N}=540\end{array}$ & $\begin{array}{l}\text { Prospective, } \\
\mathrm{N}=223\end{array}$ \\
\hline \multicolumn{5}{|l|}{ Age } \\
\hline$<21$ & $520(45.1)$ & $206(51.6)$ & $239(44.2)$ & $115(51.6)$ \\
\hline $22-24$ & $259(22.4)$ & $92(23.1)$ & $130(24.1)$ & $53(23.8)$ \\
\hline $25-31$ & $284(24.6)$ & $76(19.1)$ & $138(25.6)$ & $46(20.6)$ \\
\hline$>31$ & $91(7.9)$ & $25(6.3)$ & $33(6.1)$ & $9(4.0)$ \\
\hline \multicolumn{5}{|l|}{ Education } \\
\hline None-grade 5 & $32(2.8)$ & $7(1.8)$ & $4(0.8)$ & $0(0.0)$ \\
\hline Grades 6-9 & $181(15.7)$ & 49 (12.3) & $27(5.0)$ & $10(4.5)$ \\
\hline Grades $10-12$ & $860(74.5)$ & $333(83.5)$ & $454(84.1)$ & $188(84.3)$ \\
\hline University/college/graduate & $81(7.0)$ & $10(2.5)$ & $55(10.2)$ & 25 (11.2) \\
\hline \multicolumn{5}{|l|}{ Employment } \\
\hline No, unemployed & $1000(86.7)$ & 352 (88.2) & $463(85.7)$ & $196(87.9)$ \\
\hline Yes, part time & $78(6.8)$ & 25 (6.3) & $31(5.7)$ & $11(4.9)$ \\
\hline Yes, full time & $76(6.6)$ & $22(5.5)$ & 46 (8.5) & $16(7.2)$ \\
\hline \multicolumn{5}{|l|}{ Whom you live with } \\
\hline Alone & $45(3.9)$ & $12(3.0)$ & $25(4.6)$ & $8(3.6)$ \\
\hline Spouse & $52(4.5)$ & $15(3.8)$ & $15(2.8)$ & $8(3.6)$ \\
\hline Family/relatives & $859(74.4)$ & 315 (78.9) & $375(69.4)$ & $160(71.8)$ \\
\hline Roommate/friend & $103(8.9)$ & $34(8.5)$ & $28(5.2)$ & $12(5.4)$ \\
\hline Partner & $95(8.2)$ & $23(5.8)$ & 97 (18.0) & $35(15.7)$ \\
\hline \multicolumn{5}{|l|}{ Married } \\
\hline No & $1056(91.5)$ & $369(92.5)$ & 507 (93.9) & $211(94.6)$ \\
\hline Yes & $98(8.5)$ & $30(7.5)$ & $33(6.1)$ & $12(5.4)$ \\
\hline \multicolumn{5}{|l|}{ Contraceptive use } \\
\hline None & $540(46.9)$ & $182(45.6)$ & $157(29.1)$ & $58(26.0)$ \\
\hline Oral & $55(4.8)$ & $20(5.0)$ & $24(4.4)$ & $19(8.5)$ \\
\hline Injectable & $384(33.3)$ & $138(34.6)$ & $132(24.4)$ & $65(29.2)$ \\
\hline IUCD & $2(0.2)$ & $0(0.0)$ & $1(0.2)$ & $1(0.5)$ \\
\hline Condoms & 148 (12.9) & $52(13.0)$ & 201 (37.2) & $80(35.9)$ \\
\hline Other & $23(2.0)$ & $7(1.8)$ & $1(0.2)$ & $0(0.0)$ \\
\hline Missing & $2(0.2)$ & $0(0.0)$ & $24(4.4)$ & $0(0.0)$ \\
\hline \multicolumn{5}{|l|}{ STI signs/symptoms } \\
\hline Vaginal discharge & $155(13.4)$ & $52(13.0)$ & $154(28.5)$ & $59(26.5)$ \\
\hline Painful urination & $103(8.9)$ & $31(7.8)$ & 153 (28.3) & $57(25.6)$ \\
\hline Lower abdominal pain & $115(10.0)$ & $39(9.8)$ & $202(37.4)$ & $82(36.8)$ \\
\hline Vaginal itching & $170(14.7)$ & $60(15.0)$ & $155(28.7)$ & $63(28.5)$ \\
\hline Dyspareunia & $104(9.0)$ & $35(8.8)$ & $121(22.4)$ & $50(22.4)$ \\
\hline Vaginal sore & $27(2.3)$ & $12(3.0)$ & $142(26.3)$ & $66(29.6)$ \\
\hline \multicolumn{5}{|c|}{ No. of sex partners in last month } \\
\hline 0 & $53(4.6)$ & $18(4.5)$ & $85(15.7)$ & $40(17.9)$ \\
\hline 1 & $362(31.4)$ & $120(30.1)$ & $400(74.1)$ & $155(69.5)$ \\
\hline $2+$ & 739 (64.0) & $261(65.4)$ & 55 (10.2) & 28 (12.6) \\
\hline $\begin{array}{l}\text { Vaginal sex w/o condom past } \\
\text { week w primary partner }\end{array}$ & $638(55.3)$ & $202(50.6)$ & $190(35.2)$ & $78(35.0)$ \\
\hline $\begin{array}{l}\text { Vaginal sex w/o condom past } \\
\text { week w other partner }\end{array}$ & $150(13.0)$ & $50(12.5)$ & $8(1.5)$ & $3(1.3)$ \\
\hline
\end{tabular}


At baseline, women in the Bloemfontein study selfreported more risk behaviours than women in Rustenburg (table 1). Two-thirds of women in Bloemfontein reported vaginal intercourse with two or more partners in the month preceding cross-sectional screening; the corresponding percentage in Rustenburg was 14\%. Over half of the women in Bloemfontein reported having vaginal intercourse with their primary partners without a condom in the previous week, whereas about a quarter of women in Rustenburg reported unprotected intercourse with primary partners in the previous week. Women in Bloemfontein were also more likely to report unprotected intercourse with a non-primary partner.

The baseline features of women who entered the prospective studies were broadly similar to those of the source screening populations at both sites (table 1), and there was little indication of selection for a particular subgroup into the prospective cohort.

\section{HIV prevalence}

In Bloemfontein cross-sectional screening, the HIV prevalence was $21.2 \%$ (95\% CI $18.9 \%$ to $23.6 \%$ ), overall: $13.4 \%$ in those $18-24$ years, $38.1 \%$ in those $25-29$ years and $37.1 \%$ in women aged $30-35$ years (table 2 ). In Rustenburg, the overall HIV prevalence at screening was $23.5 \%$ (95\% CI $19.9 \%$ to $27.1 \%$ ) among women, ranging from $18.7 \%$ in those aged $18-24$ years to $31.8 \%$ in those aged $25-29$ years to $37.7 \%$ in those aged $30-35$ years (table 2).

\section{Factors associated with prevalent HIV}

Three baseline factors were significantly associated with prevalent HIV infection at both study sites (table 3): age 30 or older compared with 18-24, less versus more educational attainment and living with a child versus not living with child. Self-reported signs or symptoms of STI were associated with HIV in Bloemfontein only, and non-use of contraception versus use of condoms was associated with HIV in Rustenburg only. Sexual behaviour factors were not significantly associated with prevalent HIV infection.

\section{HIV incidence}

Nine seroconversions were observed during the prospective study in the Bloemfontein cohort, an overall incidence of 5.5/100 PY (95\% CI 2.5 to 10.4 ; table 4$)$. The incidence was $5.7 / 100 \mathrm{PY}(95 \%$ CI 2.3 to 11.7$)$ in women $18-24$ years, $4.2 / 100 \mathrm{PY}$ (95\% CI 0.1 to 23.5 ) among women 25-29 years and 5.8/100 PY (95\% CI 0.2 to 32.2 ) in the sparse $30-35$ years age stratum.

Two seroconversions were detected in the Rustenburg cohort for an overall incidence of 3.0/100 PY (95\% CI 0.4 to 10.8 ; table 4 ). Both seroconversions were in the 18-24 year age group, and the incidence in that younger age range was $4.0 / 100 \mathrm{PY}(95 \%$ CI 0.5 to 14.5$)$.

Ten of the 11 incident HIV infections at the two sites clearly occurred after enrolment in the prospective phase. One seroconversion in Bloemfontein was more problematic; rapid tests were negative at cross-sectional testing and no virus was detected in that stored specimen by PCR, but Western blot results were positive for the stored baseline specimen and were persistently blot positive until seroconversion at the 3-month visit. After excluding that Bloemfontein participant, the re-calculated Bloemfontein incidence rate was 4.9/100 PY $(95 \%$ CI 2.1 to 9.6$)$.

\section{Factors associated with incident HIV}

With nine new infections in the Bloemfontein cohort, we performed univariate proportional hazards regression with the same factors included in the analysis of risk factors for prevalent infection (above). No factor was significantly associated with incident infection. The strongest association with incident HIV was found for

\begin{tabular}{|c|c|c|}
\hline & Bloemfontein & Rustenburg \\
\hline \multicolumn{3}{|l|}{ Age $18-24$} \\
\hline Eligible population*, $\mathrm{N}$ & 776 & 369 \\
\hline HIV positive, $\mathrm{N}$ & 104 & 69 \\
\hline Prevalence $(95 \% \mathrm{Cl} \dagger)$ & $13.4 \%(11.0 \%$ to $15.8 \%)$ & $18.7 \%(14.7 \%$ to $22.7 \%)$ \\
\hline \multicolumn{3}{|l|}{ Age $25-29$} \\
\hline Eligible population*, $\mathrm{N}$ & 226 & 110 \\
\hline HIV positive, $\mathrm{N}$ & 86 & 35 \\
\hline Prevalence $(95 \% \mathrm{Cl} \dagger)$ & $38.1 \%(31.7 \%$ to $44.4 \%)$ & $31.8 \%(23.1 \%$ to $40.5 \%)$ \\
\hline \multicolumn{3}{|l|}{ Age $30+$} \\
\hline Eligible population ${ }^{*}, \mathrm{~N}$ & 143 & 61 \\
\hline HIV positive, $\mathrm{N}$ & 53 & 23 \\
\hline Prevalence $(95 \% \mathrm{Cl} \dagger)$ & $37.1 \%(29.1 \%$ to $45.0 \%)$ & $37.7 \%(25.5 \%$ to $49.9 \%)$ \\
\hline \multicolumn{3}{|l|}{ Total } \\
\hline Eligible population*, N & 1145 & 540 \\
\hline HIV positive, $\mathrm{N}$ & 243 & 127 \\
\hline Prevalence $(95 \% \mathrm{Cl} \dagger)$ & $21.2 \%(18.9 \%$ to $23.6 \%)$ & $23.5 \%(19.9 \%$ to $27.1 \%)$ \\
\hline
\end{tabular}


Table 3 Multivariable logistic regression analysis of factors associated with prevalent HIV among women screened in Bloemfontein and Rustenburg, South Africa

\begin{tabular}{|c|c|c|}
\hline Factor & $\begin{array}{l}\text { Bloemfontein } \\
\text { OR }(95 \% \mathrm{Cl})\end{array}$ & $\begin{array}{l}\text { Rustenburg } \\
\text { OR }(95 \% \mathrm{Cl})\end{array}$ \\
\hline Age $18-24$ vs $30+$ & $0.389(0.243$ to 0.623$)$ & $0.485(0.284$ to 0.826$)$ \\
\hline Age $24-29$ vs $30+$ & $1.222(0.766$ to 1.950$)$ & $0.844(0.502$ to 1.419$)$ \\
\hline Education: less than high school & 2.240 (1.552 to 3.233$)$ & 1.979 (1.099 to 3.563$)$ \\
\hline Employed: yes & $0.913(0.587$ to 1.419$)$ & $0.790(0.509$ to 1.227$)$ \\
\hline Living with child & 1.569 (1.074 to 2.290$)$ & 2.078 (1.398 to 3.088$)$ \\
\hline Married & $0.900(0.541$ to 1.498$)$ & 0.664 (0.304 to 1.450$)$ \\
\hline STI sign/symptom & 2.081 (1.472 to 2.940$)$ & 1.071 (0.729 to 1.573$)$ \\
\hline Contraception: no method vs condom/other & $1.395(0.874$ to 2.229$)$ & 2.056 (1.319 to 3.203$)$ \\
\hline Contraception: effective vs condom/other & 0.777 (0.474 to 1.273$)$ & $1.321(0.827$ to 2.111$)$ \\
\hline More than 1 partner & $1.250(0.594$ to 2.632$)$ & $1.425(0.863$ to 2.354$)$ \\
\hline 1 or more new partner & $1.168(0.853$ to 1.598$)$ & $1.136(0.724$ to 1.783$)$ \\
\hline More than 1 different partner & $0.809(0.564$ to 1.161$)$ & $0.836(0.504$ to 1.387$)$ \\
\hline 1 or more sex without condom with primary partner last month & $1.279(0.917$ to 1.786$)$ & $1.165(0.776$ to 1.748$)$ \\
\hline 1 or more sex without condom with any other partner last month & $1.067(0.622$ to 1.831$)$ & $1.224(0.444$ to 3.379$)$ \\
\hline Anal sex with primary partner & $0.828(0.407$ to 1.686$)$ & 0.974 (0.318 to 2.984$)$ \\
\hline Anal sex with any other partner & $0.660(0.183$ to 2.383$)$ & $1.333(0.153$ to 11.577$)$ \\
\hline
\end{tabular}

one or more unprotected coital acts with a non-primary partner in the last month $(\mathrm{HR}=6.7 ; \mathrm{p}=0.07)$.

\section{Pregnancy}

The prevalence of pregnancy at the time of crosssectional screening was $6.5 \%(95 \%$ CI $5.0 \%$ to $7.9 \%)$ in Bloemfontein and $8.6 \%(95 \%$ CI $6.2 \%$ to $10.9 \%)$ in Rustenburg. (Further analysis of the Rustenburg pregnancy results will appear elsewhere.) In Bloemfontein, the pregnancy prevalence in screened women ranged from $11.0 \%(95 \%$ CI $8.5 \%$ to $14.0 \%)$ in those not using a method to $3.5 \%(95 \%$ CI $1.3 \%$ to $7.5 \%)$ in condom users and $2.1 \%(95 \%$ CI $0.9 \%$ to $3.9 \%)$ in users of effective methods (hormonal and IUCDs). The Bloemfontein pregnancy incidence was 16.4/100 PY (95\% CI 10.8 to 23.9 ) and ranged from 27.6/100 PY (95\% CI 17.1 to 42.2 ) in women not using a method at baseline to 12.4/100 (95\% CI 2.6 to 36.4) in condom users and 4.7/ 100 (95\% CI 1.0 to 13.7$)$ in users of effective methods (hormonal or IUCD).

\section{DISCUSSION}

HIV prevalence in Bloemfontein's Free State Province has been close to $30 \%$ in antenatal surveillance for several years. Prevalence was lower in this study, at $21.2 \%$, yet we detected a high incidence rate of 5.5/100 PY in the overall cohort. We observed similar HIV incidence across the age spectrum in the Bloemfontein cohort, contrary to national estimates. ${ }^{8}$ The HIV prevalence among Rustenburg women in the cross-sectional screening was $23.5 \%$, again lower than recent antenatal surveillance data from the area, which could be explained by the young average age of the cohort screened. The prospective incidence rate in the Rustenburg cohort was 3.0/100 PY and higher among younger women (4.0/100 PY in 18-24 year olds).
National household survey data in South Africa show that HIV prevalence has stabilised and suggest that the incidence estimated from nationally representative surveys has declined in younger age groups. ${ }^{8}$ But the overall estimated incidence remained 1.3/100 uninfected persons of reproductive age from 2005 to $2008 .^{8}$ Others have described a pattern of stable prevalence in South African antenatal surveillance and populationbased surveys coupled with substantial incidence rates in defined cohorts. ${ }^{9}$ In the relatively few studies that have directly measured HIV incidence, it has been distressingly high in both urban and rural sites, despite multiple follow-up contacts, ongoing risk-reduction counselling and condom promotion and provision. Between 2001 and 2004, a representative cohort in one part of rural Limpopo Province had an HIV incidence of 4.9/100 PY among women, despite self-reported increases in condom use during that period. ${ }^{10}$ In the HPTN 055 cohort study, the two South African sites, both located in Kwa-Zulu Natal Province, had the highest HIV incidence rates in 2003-2004 (Durban 5.3/100 PY and Hlabisa $6.2 / 100 \mathrm{PY}) .^{11}$ In $2004-2007$, the HIV incidence rate was 6.4/100 PY and 6.5/100 PY in an urban and a rural cohort in KwaZulu-Natal, respectively. ${ }^{9}$ Three recent randomised trials of ineffective prevention products have also reported substantial HIV incidence at their South African sites. ${ }^{12-14}$ Finally, a cohort study conducted between 2007 and 2009 at two sites in Northwest Province and Western Cape Province for capacity-building purposes reported 12-month incidence rates of $6.0 / 100 \mathrm{PY}$ and $4.5 / 100 \mathrm{PY}$, similar to the rates reported here. ${ }^{15}$

Older participants had a higher risk of prevalent HIV infection in our study than younger, reflecting their longer potential for exposure to HIV. Lesser education was also associated with baseline HIV infection, as has 
Table 4 HIV incidence among women in the prospective study at the Bloemfontein and Rustenburg sites

\begin{tabular}{lll}
\hline & Bloemfontein & Rustenburg \\
\hline $\begin{array}{l}\text { Number with HIV } \\
\text { test result during } \\
\text { follow-up }\end{array}$ & 391 & 194 \\
$\begin{array}{l}\text { Confirmed HIV } \\
\text { seroconversions }\end{array}$ & 9 & 2 \\
$\begin{array}{l}\text { Person-years of } \\
\text { follow-up }\end{array}$ & 164.4 & 67.1 \\
$\begin{array}{l}\text { Incidence rate* } \\
\text { (95\% Cl†) }\end{array}$ & $5.5(2.5$ to 10.4) & $3.0(0.4$ to 10.8) \\
\hline *Per 100 person-years. & \\
tExact Cl. &
\end{tabular}

been found in other South African studies. ${ }^{10}{ }^{16}$ Other factors were not consistently associated with baseline infection across the sites, which may stem from unmeasured sexual network factors and misclassification error resulting from self-report. In fact, the striking differences in the self-reported risk behaviours between these two cohorts suggest that some self-reporting bias may have occurred. Bloemfontein participants reported high rates of multiple partnering (about two-thirds of the women) compared with national data on South African women aged $15-49$ years (3.7\% in 2008) and data on men and women in the Free State in the same 2008 survey $(14.6 \%) .{ }^{17}$ In contrast, reports of multiple partnering at the Rustenburg site were close to national averages $(12 \%-14 \%)$ and similar to a prior representative household survey in Rustenburg. ${ }^{18}$

One weakness of our analysis is that, partly due to the relatively short follow-up period, there were too few seroconversion events to do multivariable proportional hazards regression, and our univariate regression results for incident HIV infections were necessarily inconclusive. A limitation of the analysis of risk factors for prevalent HIV infections is that the behaviours reported at screening may not have reflected behaviours present at the time of infection. Lack of STI diagnostic testing precluded evaluating associations with other infections.

Perhaps the greatest study weakness relates to the validity of self-reported sexual behaviours, both for purposes of determining eligibility as well as for assessing risk during follow-up. The inaccuracies in self-reported sexual behaviour during research interviews have been amply documented and are not unique to these studies. Although we attempted to keep the eligibility factors secret from participants and outreach staff, we do not know if volunteers (both eligible and ineligible) guessed those factors and shared them with other women in the community. There may have been an awareness among women at the Bloemfontein site in particular to overreport risky behaviours to gain entry to the study: the eligibility rate was very high there and risk behaviours far exceeded national estimates. Alternatively, recruiters at the Bloemfontein, but not the Rustenburg, site may have simply tapped into a subgroup of women with particularly risky behaviour. During baseline and follow-up interviews with participants, however, we did not observe strong associations between those same behavioural factors and HIV infection.

Our study cohorts cannot be considered representative of young women in the two cities. Our behavioural eligibility criteria selected for a group of women with higher than average risk behaviours. The Rustenburg site offered the opportunity to demonstrate the impact of these selection factors: a representative household survey had been done there in 2008, 1 year before our study commenced. Our study cohort was younger, better educated, less likely to be employed and less likely to be married or live with a steady partner than all Rustenburg community residents. Most critically, only 3.4\% of women in the household survey reported multiple sexual partners in the past 3 months compared with over $10 \%$ of women screened in our study who reported multiple partners in the past 1 month. So we make no claims for representativeness: our project deliberately set out to identify and follow a cohort with higher than average risk behaviours, in order to demonstrate the suitability of the sites for future prevention research.

Countervailing study strengths include the longitudinal design with high retention rates. Classification of the HIV outcome was highly accurate, using multiple monthly rapid tests confirmed by later PCR testing. Testing of stored baseline specimens allowed us to pinpoint seroconversion and rule out acute prestudy infections. Finally, direct prospective measurement of HIV infection circumvented the lingering issues with the accuracy of cross-sectional incidence estimation.

The high HIV prevalences and incidence rates in these cohorts in two smaller South African cities should spur vigorous HIV prevention efforts. The need for HIV care and treatment in these areas is clear, and while care is available, Free State Province experienced stock-outs of HIV antiretroviral drugs during the study. ${ }^{19}$

Our results also highlight the success of the collaborative capacity-building SIDI projects: these sites are highly suitable and ready for HIV prevention research and programming. Both sites added staff and were provided broad research training, and these new skills were immediately applied in a rigorous clinical research study. Dealing with local ethics committees and community stakeholders, recruiting and enrolling potentially higher risk women, administering informed consent, bringing participants back for regular study visits, giving ongoing risk reduction and HIV counselling and making referrals for continued healthcare, all within the context of Good Clinical Practice, mimicked future prevention trials. At the conclusion of its study, JOSHA in Bloemfontein was selected to implement an oral HIV prophylaxis trial. The Aurum Institute in Rustenburg is currently conducting a trial of a vaginal microbicide, along with other behavioural HIV studies. The national research capacity has been boosted and diffused to new sites 
outside the larger cities, which can only be a boon for future HIV and other clinical research in South Africa.

Acknowledgements We thank the study participants, the study teams at The Aurum Institute and JOSHA, staff at PathCare Laboratories and at the Mangaung-University Community Partnership Program in Bloemfontein and the Departments of Health in North West and Free State Provinces. Sharne Foulkes, Ilse Reblin and Gustav Venter were vital to study success in Bloemfontein. We are also grateful to FHI 360 colleagues Lydia Mugo and Melissa Polier for clinical monitoring, Lisa Saylor for data management training and support and Sola Park for statistical support, as well as the helpful reviews of our FHI 360 colleagues and journal reviewers.

Contributors PJF, MHL, JL, CC, P-LC and CS contributed to the design of the study and its essential documents. MHL, JL and CC led data collection. PJF, MHL, JL, P-LC, CS and SF contributed to the analysis and interpretation of the data. PJF, MHL, JL, CC and SF drafted the article. All authors approved the final version of the manuscript.

Funding This work was funded by the US Agency for International Development (USAID) under Cooperative Agreement No.

GPO-A-00-05-00022-00 and the Contraceptive and Reproductive Health Technologies Research and Utilisation (CRTU) Program. Additional funding was provided by the Centers for Disease Control and Prevention (CDC) under contract no. 200-20074-05314, Task Order \#7. The contents are the responsibility of FHI 360 and do not necessarily reflect the views of USAID or the $\mathrm{CDC}$.

Competing interests None.

Ethics approval FHI 360 Protection of Human Subjects Committee; University of the Free State Ethics Committee; University of KwaZulu-Natal BREC.

Provenance and peer review Not commissioned; externally peer reviewed.

Data sharing statement No additional unpublished data will be available.

\section{REFERENCES}

1. Joint United Nations Programme on HIVIAIDS and World Health Organization. Geneva: AIDS Epidemic Update, 2009.

2. Karim QA, Karim S, Frohlich J, et al. Effectiveness and safety of tenofovir gel, an antiretroviral microbicide, for the prevention of HIV infection in women. Science 2010;329:1168-74.

3. Grant RM, Lama JR, Anderson PL, et al. Pre-exposure chemoprophylaxis for HIV prevention in men who have sex with men. N Engl J Med 2010;363:2587-99.

4. Auvert B, Taljaard D, Lagarde E, et al. Randomized, controlled intervention trial of male circumcision for reduction of HIV infection risk: the ANRS 1265 trial. PLoS Med 2005;2:e298.
5. Bailey RC, Moses S, Parker CB, et al. Male circumcision for HIV prevention in young men in Kisumu, Kenya: a randomised controlled trial. Lancet 2007;369:643-56.

6. Republic of South Africa Department of Health. National Antenatal Sentinel HIV and Syphilis Prevalence Survey in South Africa, 2009. Pretoria: Department of Health, 2010.

7. Heckathorn DD. Respondent-driven sampling: a new approach to the study of hidden populations. Soc Probl 1997;44:174-99.

8. Rehle TM, Hallett TB, Shisana O, et al. A decline in new HIV infections in South Africa: estimating HIV incidence from three national HIV surveys in 2002, 2005 and 2008. PLOS ONE 2010;5: e11094.

9. Karim QA, Kharsany ABM, Frolich JA, et al. Stabilizing HIV prevalence masks high HIV incidence rates amongst rural and urban women in KwaZulu-Natal, South Africa. Int J Epidemiol 2011;40:922-30.

10. Hargreaves JR, Bonell CP, Morison LA, et al. Explaining continued high prevalence in South Africa: socioeconomic factors, HIV incidence and sexual behavior change among a rural cohort. AIDS 2007;21(Suppl 7):S39-48.

11. Ramjee G, Kapiga S, Weiss S, et al. The value of site preparedness studies for future implementation of Phase 2/IIb/III HIV prevention trials: experience from the HPTN 055 study. J Acquir Immune Defic Syndr 2008;47:93-100.

12. Skoler-Karpoff S, Ramjee G, Ahmed K, et al. Efficacy of Carraguard for prevention of HIV infection in women in South Africa: a randomised, double-blind, placebo-controlled trial. Lancet 2008;372:1977-87.

13. Padian NS, van der Straten A, Ramjee G, et al. Diaphragm and lubricant gel for prevention of HIV acquisition in Southern African women: a randomised controlled trial. Lancet 2007;370:251-61.

14. Gray GE, Allen M, Moodie Z, et al. Safety and efficacy of the HVTN 503/Phambili Study of a clade-B-based HIV-1 vaccine in South Africa: a double-blind, randomized, placebo-controlled test-of-concept phase 2b study. Lancet Infect Dis 2011;11:507-15.

15. Nel A, Louw C, Hellstrom E, et al. HIV prevalence and incidence among sexually active females in two districts of South Africa to determine microbicide trial feasibility. PLoS One 2011;6:e21528.

16. Johnson LF, Dorrington RE, Bradshaw D, et al. The effect of educational attainment and other factors on HIV risk in South African women: results from antenatal surveillance, 2000-2005. AIDS 2009;23:1583-8.

17. Shisana O, Rehle T, Simbayi LC, et al. South African National HIV Prevalence, Incidence, Behaviour and Communication Survey 2008. A Turning Tide Among Teenagers? Cape Town: HSRC Press, 2009.

18. Latka MH, Meyer-Weitz A, Fielding K, et al. Factors Associated With Concurrent Sexual Partnering And Condom Use Are Not The Same: Results From A Representative Household Survey in Rustenburg. Durban, South Africa: Poster presented at South African AIDS Conference, 2009.

19. El-Khatib Z, Richter M. (ARV-) Free state? The moratorium's threat to patients' adherence and the development of drug-resistant HIV (letter). S Afr Med J 2009;99:412-14. 